International Tinnitus Journal. 2019;23(1):10-16.

\title{
Temporomandibular steroids in patients with tinnitus: Only on indication
}

Koning Henk ${ }^{1}$ Koning Mark²

\begin{abstract}
Introduction: Patients with tinnitus without an identifiable cause may have temporomandibular joint dysfunction and can be treated by an intra-articular injection of steroids.

Objectives: The aim of this study was to determine the efficacy of temporomandibular steroids for treating patients with tinnitus, and more specifically, to assess the parameters associated with a long-term benefit in order to improve patient selection.

Design: Subjects were 70 consecutive patients who came to our clinic from October 2016 to October 2018 for consultations on their tinnitus that persisted for one month or longer and were treated with an intra-articular injection of the temporomandibular joint with steroids. Patients charts, cervical spine radiographs and audiogram were reviewed retrospectively and data from these patients were recorded. An independent observer conducted a long-term follow-up assessment of the therapy by telephone interview.

Results: Relief of tinnitus at seven-week follow-up was achieved in $20 \%$ of the patients treated with temporomandibular steroids. At 18 months, $50 \%$ of the patients successfully treated with temporomandibular steroids for tinnitus still experienced a benefit. Adverse events of the temporomandibular steroids reported at 7 weeks of follow-up were an increase of the intensity of their tinnitus in $11 \%$ of the patients and in $3 \%$ of the patient's side-effects of the steroids. Patients with temporomandibular disorders as cause of their tinnitus were identified by the presence of unilateral tinnitus in combination with cervical pain. In patients with unilateral tinnitus together with cervical pain, $53 \%$ of them had a reduction of their tinnitus at 7 weeks following treatment with temporomandibular steroids and for $40 \%$ of them the reduction of the tinnitus was $50 \%$ or more. An increase in the intensity of their tinnitus as adverse effect occurred in $7 \%$ of the patients in this group.
\end{abstract}

Conclusions: Temporomandibular steroids can be a useful alternative for patients with tinnitus with a long term effect. However, patient selection is of vital importance, since patients with unilateral tinnitus and cervical pain respond the most to this therapy. Moreover, patient without these symptoms risk worsening the tinnitus. A prospective study should further evaluate these findings.

Keywords: Tinnitus, tempomandibular joint, tempomandibualr disorder, hearing loss, trigeminal nerve, dorsal cochlear nucleus, steroids. 


\section{INTRODUCTION}

Tinnitus is a heterogeneous condition and subtyping of different forms of tinnitus has been advocated for appropriate clinical diagnosis and management ${ }^{1}$. The term "somatic tinnitus" or "somatosensory tinnitus" is used when tinnitus appears to be related to problems of the musculoskeletal system rather than of the ear. The most common conditions in somatic tinnitus are temporomandibular joint (TMJ), head, and neck disorders. A correct diagnosis and treatment of somatic disorders underlying tinnitus play a central role for a correct management of somatic tinnitus.

Tinnitus modulation by movements of the head, neck, and jaws has been proposed as a possible indicator for somatic tinnitus ${ }^{2}$. However, the capability to modulate tinnitus itself may not indicate the presence of an underlying somatic disorder and so it cannot be used as the sole indicator for the somatic origin of tinnitus ${ }^{3}$. Valid objective diagnostic criteria of tinnitus related to TMJ disorders seem to be lacking, although it might be associated with hearing loss at lower frequencies ${ }^{4}$. A successful effect of TMJ disorder treatment on tinnitus can also be used to confirm the validity of criteria for selection of somatic tinnitus patients.

Tinnitus caused by TMJ disorders, often disappears when the temporomandibular disorder is treated successfully 5 . Intra-articular injection of steroids is a known therapeutic modality in treating painful TMJ dysfunction ${ }^{6}$. To our knowledge, the efficacy of this procedure in patients with tinnitus has never been assessed. Therefore, the aim of this study was to determine the efficacy of temporomandibular steroids injection for treating patients with tinnitus. Further aims are to find patient characteristics associated with long-term positive benefit and to test the association between low frequency hearing loss and positive effect of TMJ injection. These last two aims are performed in order to improve patient selection in which temporomandibular steroids are indicated.

\section{MATERIALS AND METHODS}

Subjects: The Medical research Ethics Committees United (Nieuwegein, the Netherlands) approved the present observational study and waived the request for informed consent for the current study. A retrospective chart review was conducted. All patients that were treated with temporomandibular steroids in our clinic between October 2016 and October 2018 for tinnitus that persisted for 1 month or longer were included. There were no exclusion criteria. All patients were previously consulted by an otorhinolaryngologist and all patients who suffered with unilateral tinnitus had anatomical pathology ruled out by MRI. The work-up of a patient with tinnitus consisted of a standardized clinical history, a bilateral audiogram and a cervical spine radiograph. The standardized clinical history included tinnitus characteristics (left side and/or right side, traumatic cause, duration of complaints and age of onset, and concomitant symptoms (self-reported hearing loss, the presence of dysbalance, dizziness, and cervicalgia). A standardized bilateral clinical audiogram assessed pure tone thresholds at 250, 500, 1000, 2000, 4000, and $8000 \mathrm{~Hz}$.

The lateral cervical spine radiographs were assessed for:

- The angle between the posterior borders of consecutive cervical vertebrae.

- The intervertebral disc space height of the five cervical levels, as assessed by the Farfan's measurement (FM): (anterior disc height + posterior disc height) / disc diameter $x 100$ per cent $^{7}$.

- The size of the anterior osteophyte relative to the size of the cervical vertebrae, as calculated by dividing the distance of the anterior border of the largest anterior osteophyte to the anterior border of the cervical vertebrae by the width of the cervical vertebrae at the middle $\times 100$ per cent ${ }^{7}$.

Treatment of temporomandibular steroids: All intraarticular injections of the temporomandibular joints were performed by an experienced anesthesiologist (HMK) in a routine manner on an outpatient basis ${ }^{8}$. The indication for these procedures was tinnitus that has lasted longer than 1 month. Each patient provided a detailed medical history and underwent a clinical examination. The intra-articular injections of the TMJ were carried out with the patient lying supine and at the side where tinnitus was present. No premedication or sedation was used. The zygomatic arch was palpated in front of the external auditory meatus. The patient was asked to open and close the mouth. The moving condyle of the mandible was palpated and marked with a marking pen. After sterile preparation, a 23-gauge needle (B. Braun Sterican single-use needle 60 $\times 25 \mathrm{~mm}$ Melsungen, Germany) was passed beneath the zygomatic arch medially, superiorly, and slightly anterior in the TMJ until bone contact was made. The needle is held in position, and the patient is asked to slowly open the mouth as a combination of $20 \mathrm{mg}$ Triamcinolone Acetonide (Kenacort Bristol-Myers Squibb, Roma, Italy) and $0.5 \mathrm{ml}$ bupivacaine $0.5 \%$ (Bupivacaine Aurobindo, Baarn, and the Netherlands) is injected. The needle is removed, and pressure applied. The patients were being observed and monitored in the recovery room for a minimal of 30 minutes and discharged home. All patients were re-evaluated by the interventionist 7 weeks after treatment.

Data Assessment: Patient charts were reviewed retrospectively to identify those who underwent a treatment with temporomandibular steroids for tinnitus. Data recorded from these patients are patient characteristics (age, sex), tinnitus characteristics (left side and/or right side, traumatic, duration of complaints, and age of onset), comorbidity (self-reported hearing loss, the presence of dysbalance, dizziness, and cervicalgia), self-reported benefit from treatment after 7 weeks (none [0\%], slight 
[less than $25 \%$ ], moderate [25\% to $50 \%$ ], good [50\% or more]), period of benefit and, if applicable, relapse. Therapy of tinnitus was continued at 7 weeks of follow-up when needed. If the patient underwent another treatment for tinnitus while he or she still had an improvement of the temporomandibular steroids, then the period of benefit from the first up until the second treatment was noted. No standardized tinnitus questionnaire was used before the procedure. A standard clinical audiogram assessed pure tone thresholds at six different frequencies from $250 \mathrm{~Hz}$ to $8 \mathrm{kHz}(250,500,1000,2000,4000$, and $8000 \mathrm{~Hz})$. Each ear was exposed to pure tones ranging from $250 \mathrm{~Hz}$ to $8 \mathrm{kHz}$ at increasing intensities until the threshold of detection was reached. The audiogram of the dominant side of the tinnitus was selected for analysis. In cases of bilateral tinnitus with equal intensity, the mean of the results of the audiogram of both sides was used. All patients with a reduction of tinnitus who received temporomandibular steroids, and who did not report any relapse, were included for an assessment by questionnaire. In December 2018, an independent observer conducted a long-term follow-up assessment of the therapy by telephone interview of the patients, using a standardized questionnaire to evaluate the current tinnitus status, the results of treatment, the time of recurrence of the original tinnitus complaints, and the need for additional therapy.

Statistical Methods: Statistical analysis was performed with Minitab 18 (Minitab Inc., State College, PA, USA). A
$\mathrm{X}^{2}$ test was used for dichotomous variables, and Student's t-test was used for continuous variables. The duration of effect of treatment was investigated using survival analysis techniques (Kaplan-Meier plot). Two multiple regression analysis were performed. One was used to yield a model from patient characteristics to predict a positive effect on tinnitus after 7 weeks from a regression model. The second multiple regression analysis tested the predictive variables of the audiogram in order to specifically evaluate the hypothesis that a positive effect of TMJ injections is predicted by low frequency hearing loss. Discriminant analysis for division in two groups was used to evaluate the correlation of the most significant variables with the self-reported benefit from temporomandibular steroids in patients with tinnitus. A value of $\mathrm{P}<0.05$ was considered statistically significant.

\section{RESULTS}

From October 2016 to October 2018, 70 patients underwent treatment with temporomandibular steroids for their tinnitus. In Table 1, the clinical characteristics of this patient are shown. Fourteen patients $(20 \%)$ responded with a reduction of their tinnitus. These responders rated the amount of relief from their tinnitus $(57 \%$ good, $14 \%$ moderate, and $29 \%$ slight). Adverse events of the temporomandibular steroids reported at 7 weeks of follow-up were an increase of the intensity of their tinnitus in $11 \%$ of the patients $(n=8)$ and in $3 \%$ of the patients side-effects of the steroids (heart palpitations, disrupted

Table 1: Clinical Characteristics of the patients with tinnitus.

\begin{tabular}{|c|c|c|c|}
\hline & Prevalence & Median & Q1 - Q3 \\
\hline Age at tinnitus onset (year) & & 57 & $49.8-64.3$ \\
\hline Gender (male) & $54 \%$ & & \\
\hline Unilateral tinnitus & $39 \%$ & & \\
\hline Self-perceived hearing loss & $60 \%$ & & \\
\hline Cervical pain & $71 \%$ & & \\
\hline Period of tinnitus (year) & & 5.5 & $2.0-17.0$ \\
\hline Painfull jaw joint on physical examination & $17 \%$ & & \\
\hline \multicolumn{4}{|l|}{ Hearing loss (dB) at: } \\
\hline $250 \mathrm{~Hz}$ & & 15 & $10.0-27.5$ \\
\hline $500 \mathrm{~Hz}$ & & 15 & $10.0-25.0$ \\
\hline $1 \mathrm{KHz}$ & & 15 & $10.0-30.0$ \\
\hline $2 \mathrm{KHz}$ & & 20 & $10.0-35.0$ \\
\hline $4 \mathrm{KHz}$ & & 40 & $20.0-55.0$ \\
\hline $8 \mathrm{KHz}$ & & 40 & $25.0-63.8$ \\
\hline Angle between vertebrae C2 and C6 (degrees) & & 6 & $0.8-14.3$ \\
\hline \multicolumn{4}{|l|}{ Farfan's measurement of disc space height (\%): } \\
\hline $\mathrm{C} 2-\mathrm{C} 3$ & & 40.5 & $35.8-44.0$ \\
\hline C3-C4 & & 37 & $29.0-44.0$ \\
\hline C4-C5 & & 37.5 & $30.0-41.0$ \\
\hline $\mathrm{C} 5-\mathrm{C} 6$ & & 28 & $20.0-35.5$ \\
\hline $\mathrm{C} 6-\mathrm{C} 7$ & & 26 & $21.0-34.0$ \\
\hline \multicolumn{4}{|l|}{ Size of anterior osteophyte (\%) at: } \\
\hline C3 & & 6 & $0.0-10.3$ \\
\hline $\mathrm{C} 4$ & & 9 & $6.0-14.0$ \\
\hline C5 & & 16 & $13.0-21.0$ \\
\hline C6 & & 12 & $8.0-16.0$ \\
\hline
\end{tabular}

DB: Decibel; Hz: Hertz, KHz: Kilohertz. Q1-Q3: Inter-Quartile Range. 
menstrual bleeding; $n=2$ ). Figure 1 shows a Kaplan-Meier plot representing the probability of sustained tinnitus relief after temporomandibular steroids in patients with tinnitus who were treated successfully $(n=14)$. The time to followup assessment varied from 2 months to 24 months, with a median time of 18 months. At 18 months, $50 \%$ of the patients successfully treated with temporal steroids for tinnitus still had a benefit. Patients with a positive effect of temporomandibular steroids on the tinnitus at 7 weeks were compared with the non-responders (Table 2). The presence of cervical pain and the size of the anterior osteophyte at the vertebrae $\mathrm{C} 5$ had a statistically significant association with improvement by treatment. Multivariate statistical analysis with variables of the clinical history, audiogram and the cervical spine radiographs indicated that the presence of cervical pain in combination with the

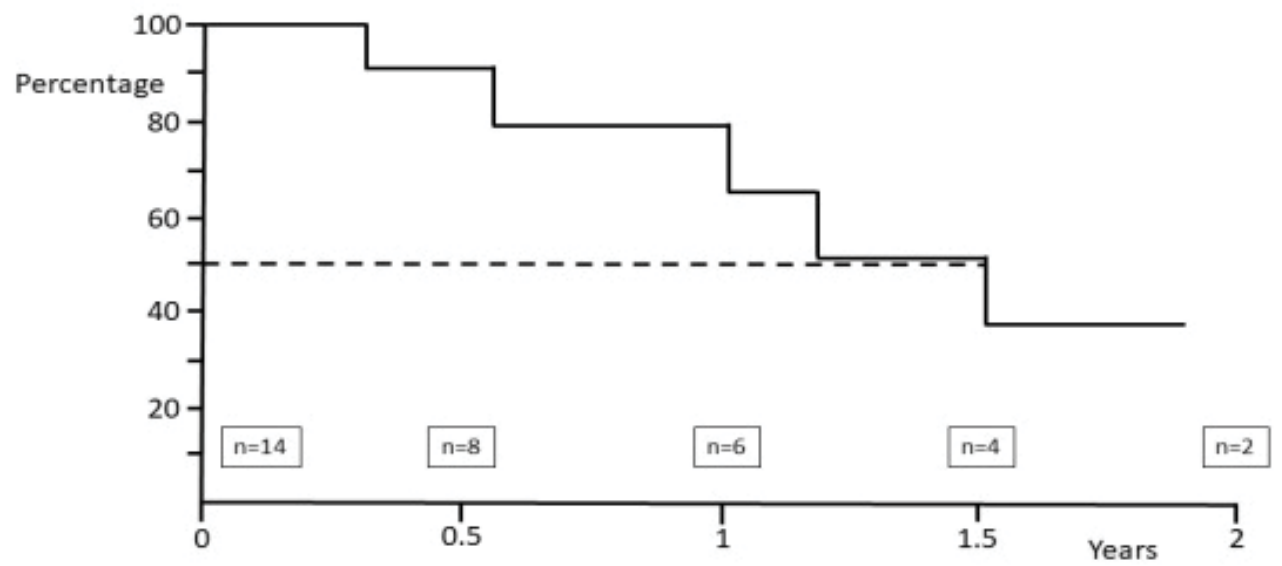

Figure1: Kaplan-Meier plot to show probability of sustained tinnitus relief in successfully treated patients $(n=14)$ after an infiltration with steroids of the temporomandibular joint.

Table 2: Patients with a positive effect of Temporomandibular steroids injection on their tinnitus at 7 weeks were compared with the non-responders.

\begin{tabular}{|c|c|c|c|c|c|c|c|c|}
\hline & \multicolumn{3}{|c|}{$\begin{array}{l}\text { Positive effect of temperomandibular } \\
\text { steroids }(n=14)\end{array}$} & \multicolumn{3}{|c|}{$\begin{array}{l}\text { No effect of temperomandibular } \\
\text { steroids }(n=56)\end{array}$} & \multirow[t]{2}{*}{ P-value } & \\
\hline & Prev. & Mean & SEM & Prev. & Mean & SEM & & \\
\hline Age at tinnitus onset (year) & & 47.1 & 3.4 & & 44.4 & 1.9 & 0.498 & \\
\hline Gender (male) & $36 \%$ & & & $59 \%$ & & & 0.119 & \\
\hline Unilateral tinnitus & $57 \%$ & & & $34 \%$ & & & 0.110 & \\
\hline Self-perceived hearing loss & $50 \%$ & & & $63 \%$ & & & 0.393 & \\
\hline Cervical pain & $93 \%$ & & & $66 \%$ & & & 0.047 & Sign. \\
\hline Period of tinnitus (year) & & 7.38 & 2.0 & & 11.7 & 1.9 & 0.123 & \\
\hline $\begin{array}{l}\text { Painfull jaw joint on physical } \\
\text { examination }\end{array}$ & $29 \%$ & & & $14 \%$ & & & 0.201 & \\
\hline \multicolumn{9}{|l|}{ Hearing loss (dB) at: } \\
\hline $250 \mathrm{~Hz}$ & & 34.5 & 8.5 & & 20 & 2.6 & 0.128 & \\
\hline $500 \mathrm{~Hz}$ & & 31.1 & 7.7 & & 19.6 & 2.8 & 0.186 & \\
\hline $1 \mathrm{KHz}$ & & 29.2 & 8.5 & & 22.2 & 2.9 & 0.451 & \\
\hline $2 \mathrm{KHz}$ & & 26.7 & 8.5 & & 24.5 & 2.7 & 0.812 & \\
\hline $4 \mathrm{KHz}$ & & 37.5 & 7.9 & & 40.5 & 3.2 & 0.731 & \\
\hline $8 \mathrm{KHz}$ & & 45.5 & 3.5 & & 37.3 & 6.6 & 0.286 & \\
\hline $\begin{array}{l}\text { Angle between vertebrae C2 and C6 } \\
\text { (degrees) }\end{array}$ & & 9.9 & 2.6 & & 6.9 & 1.4 & 0.319 & \\
\hline \multicolumn{9}{|c|}{ Farfan's measurement of disc space height (\%): } \\
\hline $\mathrm{C} 2-\mathrm{C} 3$ & & 40.6 & 1.8 & & 39.5 & 1.0 & 0.615 & \\
\hline C3-C4 & & 32.7 & 2.6 & & 36.8 & 1.2 & 0.178 & \\
\hline C4-C5 & & 37.6 & 2.4 & & 35 & 1.1 & 0.343 & \\
\hline C5-C6 & & 26.4 & 2.5 & & 28.7 & 1.3 & 0.419 & \\
\hline $\mathrm{C} 6-\mathrm{C} 7$ & & 28.5 & 2.8 & & 27.3 & 1.3 & 0.698 & \\
\hline \multicolumn{9}{|l|}{ Size of anterior osteophyte (\%) at: } \\
\hline C3 & & 7.5 & 1.8 & & 5.7 & 0.8 & 0.355 & \\
\hline $\mathrm{C} 4$ & & 10.1 & 0.6 & & 10.9 & 1.3 & 0.605 & \\
\hline C5 & & 18.6 & 1.0 & & 15.5 & 1.1 & 0.045 & Sign. \\
\hline C6 & & 13.1 & 2.1 & & 12.6 & 1.0 & 0.830 & \\
\hline
\end{tabular}

DB: Decibel; Hz: Hertz, KHz: Kilohertz; SEM: Standard Error of the Mean; Sign: Significant; Prev: Prevalence 
presence of unilateral tinnitus could predict a positive effect of temporomandibular steroids on the tinnitus at 7 weeks the best (Proportion Correct $=0.814$ ). In patients with unilateral tinnitus together with cervical pain, $53 \%$ of them had a reduction of their tinnitus at 7 weeks following treatment with temporomandibular steroids and in $40 \%$ of them the reduction of the tinnitus was $50 \%$ or more (Table $3)$. An increase in the intensity of their tinnitus as adverse effect occurred in $7 \%$ of the patients in this group.

Unilateral tinnitus in combination with cervical pain has a sensitivity of $57 \%$ and a specificity of $87.5 \%$ in predicting a good response to temporomandibular steroids in patients with tinnitus. The positive and negative predictive values were $53 \%$ and $89 \%$ respectively.

Again multivariate statistical analysis was performed with only variables of the audiogram. The variables hearing loss at $250 \mathrm{~Hz}$ and at $1000 \mathrm{~Hz}$ were significant and could predict a positive effect of temporomandibular steroids on tinnitus at 7 weeks follow-up (Proportion Correct=0.75) (Figure 2).

\section{DISCUSSION}

In a cohort of patients with tinnitus that persisted for one month or longer, $20 \%$ of the patients responded with a reduction of their tinnitus after an intra-articular injection of the TMJ with steroids. At 18 months, $50 \%$ of the initially successful treated patients still experienced a benefit. Adverse events of the temporomandibular steroids at 7 weeks of follow-up were an increase of the intensity of the tinnitus in $11 \%$ of the patients and in $3 \%$ of the patient's side-effects of the steroids. The presence of cervical pain and unilateral tinnitus and hearing loss at $250 \mathrm{~Hz}$ and $1000 \mathrm{~Hz}$ were associated with a positive treatment effect.

A correct diagnosis and treatment of somatic disorders underlying tinnitus play a central role for a correct management of somatic tinnitus ${ }^{1}$. However, the identification of somatic tinnitus may be complex. Among individuals with somatic tinnitus, a high proportion reported variation in the loudness of their tinnitus, and the presence of TMJ disorders ${ }^{9}$. TMJ disorders are characterized by joint pain, joint dysfunction, joint noises, tension headaches and tinnitus ${ }^{10}$. Habits of bruxism or clenching, unilateral mastication and maxillofacial injury history may be risk factors of TMJ disorders ${ }^{11}$. Tinnitus can also be modulated by specific manoeuvres of the temporomandibular joint, head and neck. However, the capability to modulate tinnitus itself may not indicate the presence of an underlying somatic disorder ${ }^{3}$.

TMJ disorders associated with tinnitus is considered to be a subgroup with specific therapeutic management ${ }^{12,13}$. Dental functional therapy, stomatognathic therapy, intraoral

Table 3: The presence of unilateral tinnitus in combination cervical pain predicts a positive effect of temporomandibular steroids on the tinnitus at 7 weeks.

\begin{tabular}{|c|c|c|c|c|c|c|}
\hline Group & Cervical pain & Tinnitus & Positive effect & $\begin{array}{c}\text { Self-reported benefit of } \\
50 \% \text { or more }\end{array}$ & Prevalence & $\begin{array}{l}\text { An increase of tinnitus } \\
\text { following therapy }\end{array}$ \\
\hline 0 & No & Bilateral & $12.50 \%$ & $0 \%$ & $12 \%$ & $0 \%$ \\
\hline 1 & No & Unilateral & $0 \%$ & $0 \%$ & $17 \%$ & $20 \%$ \\
\hline 2 & Yes & Bilateral & $14.30 \%$ & $5.70 \%$ & $50 \%$ & $13 \%$ \\
\hline 3 & Yes & Unilateral & $53 \%$ & $40 \%$ & $21 \%$ & $7 \%$ \\
\hline
\end{tabular}

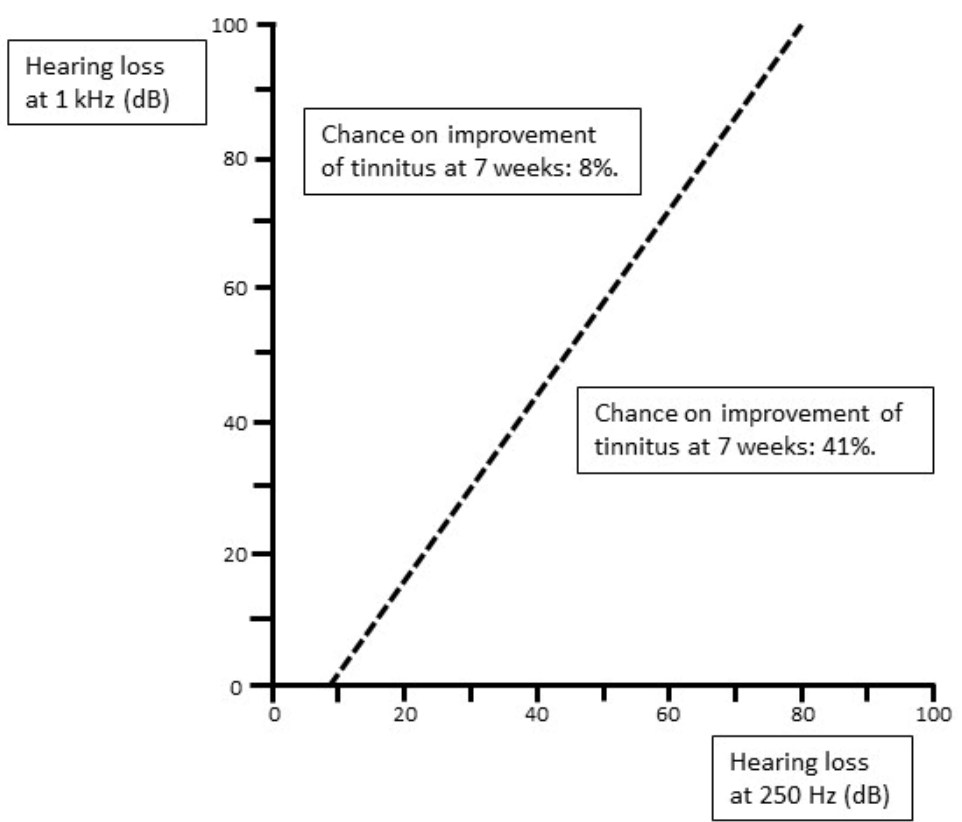

Figure 2: Hearing loss at $250 \mathrm{~Hz}$ and at $1000 \mathrm{~Hz}$ can predict a positive effect of temporomandibular steroids on tinnitus at 7 weeks follow-up (Proportion Correct $=0.75$ ). For instance, if a patient has $60 \mathrm{~dB}$ hearing loss at $250 \mathrm{~Hz}$ and $20 \mathrm{~dB}$ hearing loss at $1 \mathrm{kHz}$, there is a $41 \%$ chance of improvement. 
splint therapy, and acupuncture were reported to have a favourable effect on TMJ disorder-related tinnitus ${ }^{5-14}$. Also, temporomandibular steroids have been mentioned as therapeutic option ${ }^{15}$. The overall effect of TMJinjection of steroids was limited in this cohort. However, temporomandibular steroids were effective in patients with unilateral tinnitus and cervical pain. Moreover, an increase in the intensity of their tinnitus occurred the least in this specific group. Conversely, patients without these two symptoms had minimal response, but an increase in the intensity of tinnitus occurred more often. Therefore, patient selection seems critical besides the cervical pain and unilateral tinnitus, this study found hearing loss at 250 $\mathrm{Hz}$ and $1000 \mathrm{~Hz}$ to be predictive for a positive result of TMJ injection.

The mechanism in which TMJ dysfunction is related with tinnitus is believed to involve the dorsal cochlear nucleus, since the somatosensory input of the TMJ is transmitted through branches of the mandibular division of the trigeminal nerve to the ventral cochlear nucleus, dorsal cochlear nucleus and inferior colliculus ${ }^{16-18}$. Trigeminal stimulations have an influence on the activity of the dorsal cochlear nucleus, especially in case of an associated hearing loss ${ }^{19}$. Deafness increases the spontaneous rates of the somatosensory neurons of the trigeminal nerve and results in a greater sensitivity of the dorsal cochlear nucleus to trigeminal stimulation ${ }^{20,21}$. A majority of patients with TMJ dysfunction have hearing impairment at low frequencies ${ }^{4}$. Hearing loss at low frequencies might explain the fact that only some patients with TMJ dysfunction will experience tinnitus. When the relationship between TMJ disorders and tinnitus is studied, we recommend performing an audiogram in the subjects, to further specify the hearing loss.

This study has several limitations. First, the current retrospective study comes with inherent limitations. A prospective follow-up study would be necessary to confirm these results. Second limitation is the number of patients in this study. Even though these numbers are sufficient to demonstrate an effect in this explorative study, re-evaluation of these predictors is necessary in a larger cohort. The third limitation could be the use of selfreported benefit as outcome measure for therapy instead of standardized tinnitus questionnaires. In a prospective follow-up study adding such a questionnaire could give more information for the success of therapy. The fourth limitation could be the use of exclusion criteria in this study. However, one of the aims of our study was to find patient characteristics associated with long-term positive benefit in order to improve patient selection in which temporomandibular steroids are indicated. Exclusion criteria could cause a bias and therefore it was rejected.

\section{CONCLUSION}

Temporomandibular steroids can be a useful alternative for patients with tinnitus with a long term effect. However, patient selection is of vital importance, since patients with unilateral tinnitus and cervical pain respond the most to this therapy. Moreover, patient without these symptoms risk worsening the tinnitus. A prospective study should further evaluate these findings.

\section{CONFLICT OF INTEREST}

The Author declares no conflict of interest

\section{REFERENCES}

1. Ralli M, Greco A, Turchetta R, Altissimi G, de Vicentiis M, Cianfrone G. Somatosensory tinnitus: Current evidence and future perspectives. J Int Med Res. 2017;45:933-47.

2. Ralli M, Greco A, Boccassini A, Altissimi G, Di Paolo C, Falasca V, et al. Sub-typing patients with somatic tinnitus: Modulation of tinnitus and history for somatic dysfunction help identify tinnitus patients with temporomandibular joint disorders. Plos one. 2018;13:e0202050.

3. Levine RA, Nam EC, Oron Y, Melcher JR. Evidence for a tinnitus subgroup responsive to somatosensory based treatment modalities. Prog Brain Res. 2007;166:195-207.

4. Pekkan G, Aksoy S, Hekimoglu C, Oghan F. Comparative audiometric evaluation of temporomandibular disorder patients with otological symptoms. J. Craniomaxillofac. Surg. 2010;38:231-4.

5. Wright EF, Syms CA, Bifano SL. Tinnitus, dizziness, and nonotologic otalgia improvement through temporomandibular disorder therapy. Mil Med. 2000; 165:733-6.

6. Isacsson G, Schumann M, Nohlert E, Mejersjö C, Tegelberg A. Pain relief following a single-dose intra-articular injection of methylprednisolone in the temporomandibular joint arthralgia- $A$ multicentre randomised controlled trial. J oral rehabil. 2019;46:5-13.

7. Koning HM, Koning MV, Koning NJ, Ter Meulen BC. Anterior Cervical Osteophytes and Sympathetic Hyperactivity in Patients with Tinnitus: Size Matters. Int. Tinnitus J. 2018;22:97-102.

8. Hammer M, Noe CE, Racz GB. Joint blocks of the Head and Neck. In: Prithvi Raj P. edition Interventional Pain management. ImageGuided Procedures. $2^{\text {nd }}$ edition Philadelphia. The United States of America: Saunders Elsevier. 2008:189-91.

9. Ward J, Vella C, Hoare DJ, Hall DA. Subtyping somatic tinnitus: A cross-sectional UK cohort study of demographic, clinical and audio logical characteristics. PLoS One 2015;10:e0126254.

10. Moreno-Fernández AM, Jiménez-Castellanos E, Iglesias-Linares A, Bueso-Madrid D, Fernández-Rodríguez A, de Miguel $M$. Fibromyalgia syndrome and temporomandibular disorders with muscular pain. A review. Mod Rheumatol. 2017;27(2):210-6.

11. Hu XX, Zhu YM, He LT, Gu Y, Liang ZG, Zheng CS. Investigation of related risk factors of temporomandibular disorders in 109 patients. Sanghai j Stomatology. 2017;26(2):213-6.

12. Algieri GMA, Leonardi A, Arangio P, Vellone V, Paolo CD, Cascone P. Tinnitus in Temporomandibular Joint Disorders: Is it a Specific Somatosensory Tinnitus Subtype.Int. Tinnitus J. 2017;20(2):83-7.

13. Vielsmeier V, Strutz J, Kleinjung T, Schecklmann M, Kreuzer PM, Landgrebe $\mathrm{M}$, et al. Temporomandibular joint disorder complaints in tinnitus: further hints for a putative tinnitus subtype. PLoS One. 2012;7(6):e38887.

14. Strom D, Behrenth E, Ekman K, Johansson A, Unell L, Carlsson GE: Management of tinnitus and jaw-muscle tenderness using an intraoral appliance and acupuncture. Swed Dent J. 2013;37(3):105-10.

15. Kleinjung T. Surgical Treatments: Introduction. In: Møller AR, Langguth B, De Ridder D, Kleinjung T eds. Textbook of Tinnitus. First edition. New York, United States of America: Springer. 2011:661.

16. Paparo F, Fatone FM, Ramieri V, Cascone P. Anatomic relationship between trigeminal nerve and temporomandibular joint. Eur Rev Med Pharmacol Sci. 2008;12(1):15-8. 
17. Zhou J, Shore S. Projections from the trigeminal nuclear complex to the cochlear nuclei: A retrograde and anterograde tracing study in the guinea pig. J Neurosci Res. 2004;78(6):901-7.

18. Zhou J, Shore S. Convergence of spinal trigeminal and cochlear nucleus projections in the inferior colliculus of the guinea pig. $J$ Comp Neurol. 2006;495(1):100-12.

19. Shore SE, Koehler S, Oldakowski M, Hughes LF, Syed S. Dorsal cochlear nucleus responses to somatosensory stimulation are enhanced after noise-induced hearing loss. Eur $\mathrm{J}$ Neurosci. 2008;27(1):155-68.

20. Dehmel S, Cui YL, Shore SE. Cross-modal interactions of auditory and somatic inputs in the brainstem and midbrain and their imbalance in tinnitus and deafness. Am $\mathrm{J}$ Audiol. 2008;17(2):S193-209.

21. Zeng C, Yang Z, Shreve L, Bledsoe S, Shore S. Somatosensory projections to cochlear nucleus are up regulated after unilateral deafness. J Neurosci. 2012;32(45):15791-801. 